\title{
传统光滑粒子动力学方法的适用性分析
}

\author{
周光正 ${ }^{*}$, 葛蔚, 李静海 \\ 中国科学院过程工程研究所, 多相复杂系统国家重点实验室, 北京 100190 \\ * 联系人, E-mail: gzzhou@home.ipe.ac.cn \\ 2013-01-25 收稿, 2013-04-09 接受 \\ 国家自然科学基金(21206167)和中国科学院战略性先导科技专项(XDA07080203)资助
}

\begin{abstract}
摘要 光滑粒子动力学 (smoothed particle hydrodynamics, SPH) 是一种典型的无网格拉格朗 日型数值方法, 但它存在计算精度相对较低的缺陷. 为调查精度问题的物理本质, 将 SPH 粒 子类比于实体物理颗粒, 分析了 SPH 粒子的弹性效应, 并提出了一个表征黏弹效应比的无量 纲数. 通过二维 Couette 流模拟, 考察了该无量纲数对粒子速度剖面与排布方式的影响. 研究 结果表明, 系统的有效秥度随该无量纲数的增大而减小, 且粒子数的增加只能显著降低较小 无量纲数下的有效黏度. 名义黏性耗散、总耗散以及理论耗散之间的差异, 进一步验证了弹 性效应所导致的非物理耗散. 总之, 由于马赫数与该无量纲数的限制, SPH 模拟存在一个临 界雷诺数，只有低于这个临界数，才能基于合适的模型参数实现牛顿流体的精确模拟.
\end{abstract}

关键词

光滑粒子动力学 流体力学

精度

Couette 流

粒子方法
复杂流动的机理研究对很多科学和工程问题至 关重要, 数值模拟在这方面扮演越来越重要的角色. 光滑粒子动力学 (smoothed particle hydrodynamics, $\mathrm{SPH})$ 是一种典型的无网格粒子方法, 它在处理具有 复杂边界或变形界面的流动问题上具有独特优势. 类似于其他粒子方法, SPH 将连续介质离散为大量具 有物理属性的数值粒子. 流体动力学守恒方程被 $\mathrm{SPH}$ 离散算法转化为类似于分子动力学 (molecular dynamics, MD) 描述的常微分方程. SPH 粒子基于这 些方程与邻域粒子相互作用, 并不断更新每一时刻 的位置. 相对于网格类方法的复杂界面追踪或捕捉 技术, 如水平集(level set method)、标记网格法(marker and cell, MAC)、流体体积法(volume of fluid, VOF)等, SPH 直接通过粒子的分布状况确定相界面, 且与所 含流体相数完全无关. SPH 最初应用于天体物理问 题 ${ }^{[1,2]}$, 目前已拓广到流体动力学甚至固体力学的多 个领域, 包括自由表面流 ${ }^{[3,4]}$ 、多相流 ${ }^{[5,6]}$ 、非牛顿 流 $^{[7,8]}$ 、湍流 ${ }^{[9,10]}$ 以及流固耦合 ${ }^{[11]}$ 等.

然而, 传统 SPH 方法存在一些固有缺陷, 包括
相对较低的计算精度、张力不稳定性以及不易处理边 界条件 ${ }^{[12]}$. 在数值精度的理论分析方面, SPH 的一致 性问题已经被广泛研究 ${ }^{[13,14]}$. 本质上, 由于 SPH 粒子 的自适应特征, 不规则的粒子分布状态导致 SPH 不 满足变量及其导数近似的一致性条件 ${ }^{[13,15]}$. 目前, 已 存在多种改进传统 SPH 方法的技术方案. 再生核粒 子法(reproducing kernel particle method, PKPM) ${ }^{[16]}$ 通 过构建校正函数改善 SPH 的一致性问题. 基于泰勒 级数展开, Chen 等人 ${ }^{[17]}$ 与 $\mathrm{Liu}$ 等人 ${ }^{[18]}$ 分别开发了针 对固体力学与流体力学的修正光滑粒子法 (corrective smoothed particle method, CSPM)与有限粒子法(finite particle method, FPM). Jiang 等人 ${ }^{[19]}$ 甚至提出了 mixed corrected symmetric SPH (MC-SSPH), 该方法 在多种问题上皆表现出较高的精度与稳定性. 此外, 移动最小二乘粒子动力学(moving-least-squares-particle hydrodynamics, MLSPH) ${ }^{[20]}$ 采用伽辽金方法(Galerkin method), 并定义了与热力学相容的新粒子体积. 为 提高密度场与压力场的计算精度, Chaniotis 等人 ${ }^{[21]}$ 使用重新网格化策略, 通过对粒子位置的周期性初 013-5889-9 
始化，人工恢复粒子分布状态的规则性.

总体而言, 绝大多数 SPH 改进方案的数值实现 都很复杂, 而且具有较大的计算量. 此外, 一些 SPH 改进方案甚至需要借助背景网格, 这使之失去了粒 子方法极具吸引力的无网格特征. 由于概念的简洁 与数值实现的便捷, 传统 SPH 方法一直以来应用最 为广泛. 另一方面, Monaghan ${ }^{[22]}$ 提出粒子方法本质 上与流体的微观或介观物理具有某种意义的内在联 系. 事实上, 已有研究深人分析了绝热理想气体无黏 流动的 SPH 模拟与分子动力学描述在多方面的相似 性 ${ }^{[23,24]}$. 针对复杂流体, Español 和 Revenga ${ }^{[25]}$ 甚至提 出了所谓的光滑耗散粒子动力学 (smoothed dissipative particle dynamics, SDPD), 该方法在 SPH 理论的 基础上以一种热力学相容的方式耦合热脉动. 另外, 基于表面张力的微观物理起源, 在所有粒子间进一 步增添某种形式的引力 ${ }^{[26,27]}$, 或仅在不同相粒子间 施加合理的斥力 ${ }^{[5,28,29]}$, 皆可实现表面张力的 SPH 模 拟. 这些现象表明, SPH 粒子确实与微观物理粒子具 有某种相似性, SPH 的简单公式也远不只是宏观流动 的数学描述.

对于不可压缩流动的 SPH 模拟, 虽然存在一些 指导准则, 但理论并不严谨, 相关参数的选择有一定 的经验性. 众所周知, 无量纲数对不同系统的流型分 析具有重要作用, 如雷诺数、弗劳德数、韦伯数等. 因 此, 从传统 SPH 方法内在无量纲数的角度分析该方 法的物理机理与适用性, 具有重要意义. 在前期工作的 基础上 ${ }^{[30]}$, 本文提出了一个表征黏弹效应比的无量 纲数, 并考察了该无量纲数对系统多方面性质的影 响. 研究结果表明, 在某种程度上, 该无量纲数可作 为评估 SPH 模拟相对牛顿流体偏差程度的判据.

\section{1 数值方法}

\subsection{SPH 基本理论}

$\mathrm{SPH}$ 本质上是基于插值理论. 该方法以离散的 形式, 将待求函数 $f(\boldsymbol{r})$ 在任意点 $\boldsymbol{r}$ 的值表示为其邻域 粒子的总和

$$
f(\boldsymbol{r})=\sum_{j} \frac{m_{j}}{\rho_{j}} f\left(\boldsymbol{r}_{j}\right) W\left(\boldsymbol{r}-\boldsymbol{r}_{j}, h\right),
$$

式中 $m_{j}, \rho_{j}$ 与 $\boldsymbol{r}_{j}$ 分别是粒子 $j$ 的质量、密度与位置; $W$ 是权函数, 其光滑长度 $h$ 决定它的有效范围. 类似地, 函数的梯度 $\nabla f(\boldsymbol{r})$ 的计算式为

$$
\nabla f(\boldsymbol{r})=\sum_{j} \frac{m_{j}}{\rho_{j}} f\left(\boldsymbol{r}_{j}\right) \nabla W\left(\boldsymbol{r}-\boldsymbol{r}_{j}, h\right) .
$$

权函数必须在光滑长度趋向于零时具有狄拉克函数 性质，而且满足正则化条件

$$
\int W\left(\boldsymbol{r}-\boldsymbol{r}^{\prime}, h\right) \mathrm{d} \boldsymbol{r}^{\prime}=1 .
$$

鉴于权函数的二阶连续性对 SPH 的稳定性至关重要, 本文采用五次样条函数的二维表达式 ${ }^{[31]}$.

根据式(1), 粒子密度的计算式为

$$
\rho_{i}=\sum_{j} m_{j} W\left(\left|\boldsymbol{r}_{i}-\boldsymbol{r}_{j}\right|, h\right) .
$$

式(4)在理论上严格保证流体的质量守恒. 基于满足 变分一致性的压力梯度对称格式与来源于积分近似 的黏性耗散项表达式,一个较流行的 N-S 方程离散 形式为 ${ }^{[26,31]}$

$$
\begin{aligned}
\frac{\mathrm{d} \boldsymbol{v}_{i}}{\mathrm{~d} t}= & -\sum_{j} m_{j}\left(\frac{P_{i}}{\rho_{i}^{2}}+\frac{P_{j}}{\rho_{j}^{2}}\right) \nabla_{i} W_{i j} \\
& +\sum_{j} \frac{m_{j}\left(\mu_{i}+\mu_{j}\right)\left(\boldsymbol{v}_{i}-\boldsymbol{v}_{j}\right)}{\rho_{i} \rho_{j}}\left(\frac{1}{r_{i j}} \frac{\partial W_{i j}}{\partial r_{i}}\right)+\boldsymbol{g},
\end{aligned}
$$

式中 $P_{i}, \mu_{i}$ 与 $v_{i}$ 分别是粒子 $i$ 的压力、动力黏度与速 度, $r_{i j}$ 是粒子 $i$ 与 $j$ 之间的距离, $W_{i j}=W\left(\boldsymbol{r}_{i}-\boldsymbol{r}_{i}, h\right), \nabla_{i}$ 表示相对粒子 $i$ 坐标的梯度. 式(5)中的下标 $i$ 代表任 意流体粒子, 而下标 $j$ 代表 $i$ 粒子的邻域粒子, 它包 括流体粒子与边界粒子.

对于不可压缩流动的模拟, 流体的真实状态方 程通常要求极小的时间步长. 因此, 一般采用人工状 态方程进行近似模拟。一个典型的简单状态方 程 ${ }^{[27,31,32]}$ 为

$$
P=c^{2} \rho=K \rho,
$$

式中 $c$ 是数值声速, $K$ 是 “刚度参数”. 通常利用较大 的数值声速保证足够小的流体密度变化. 根据 Monaghan ${ }^{[3]}$ 的观点, 流体密度相对变化率 $\delta$ 与马赫数 $\mathrm{Ma}$ 的近似关系为

$$
\delta=\frac{\Delta \rho}{\rho_{0}} \approx \mathrm{Ma}^{2}=\left(\frac{V_{\max }}{c}\right)^{2}=\frac{V_{\max }^{2}}{K},
$$

式中 $V_{\max }$ 是最大流场速度. Morris 等人 ${ }^{[31]}$ 认为, $\delta$ 应 该小于 3\% (一般为 1\%)才能保证较高的压力场计算 精度.

\section{2 边界条件}

相对基于网格的数值方法, SPH 在固壁边界的实 
施上更为复杂. 大体上说, 固壁处理主要有 3 种方案, 它们分别采用排斥力、镜像粒子以及边界粒子. 排斥 力法由 Monaghan ${ }^{[3,22]}$ 提出, 它直接在固壁表面上布 置一层粒子, 该粒子将对与之重叠的流体粒子施加 强大的排斥力. 虽然该方法可以有效阻止流体进人 固壁, 但固壁附近流体粒子的支持域却缺少足够多 的粒子. 镜像粒子法 ${ }^{[33,34]}$ 通过流体粒子沿固壁表面 的镜像映射引人镜像粒子, 但这种技术不易处理复 杂几何边界. 边界粒子法则直接向固壁区域延展流 体粒子的初始规则分布状态, 从而获得数层相对固 定的边界粒子 ${ }^{[31,35,36]}$. 这些边界粒子参与邻域流体粒 子的相关计算, 而且该技术相对镜像粒子法具有较 小的计算量.

此外, SPH 通常采用对边界粒子赋予虚拟速度的 方式实现边界无滑移条件. 如图 1 所示, 根据固壁附 近流场满足速度线性分布的假设 ${ }^{[31,35]}$, 当速度为 $\boldsymbol{v}_{i}$ 的流体粒子 $i$ 与边界粒子 $j$ 作用时, 粒子 $j$ 的虚拟速 度为

$$
\boldsymbol{v}_{j}=\boldsymbol{V}+\frac{d_{j}}{d_{i}}\left(\boldsymbol{V}-\boldsymbol{v}_{i}\right),
$$

式中 $d_{i}$ 与 $d_{j}$ 分别为粒子 $i$ 与 $j$ 到固壁表面的法向距离, $\boldsymbol{V}$ 是固壁的速度. 若固壁静止, 式(8)进一步简化为

$$
\boldsymbol{v}_{j}=-\frac{d_{j}}{d_{i}} \boldsymbol{v}_{i} .
$$

同时，一般假定固壁满足人工 Neumann 压力条 件 ${ }^{[10,34]}$.

\section{3 黏弹效应比}

当把式(5)中的粒子压力与黏度分别用人工状态 方程(式(6)) 与流体动力黏度 $\left(\mu_{0}\right)$ 替换, 并且忽略其中 的重力项, 式(5)转变为如下形式

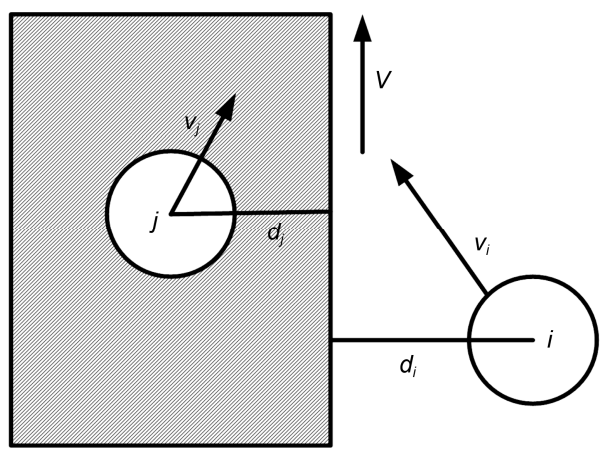

图 1 无滑移边界条件示意图

$$
\begin{aligned}
\frac{\mathrm{d} \boldsymbol{v}_{i}}{\mathrm{~d} t}= & -K \sum_{j} m_{j}\left(\frac{1}{\rho_{i}}+\frac{1}{\rho_{j}}\right) \nabla_{i} W_{i j} \\
& +2 \mu_{0} \sum_{j} \frac{m_{j}\left(\boldsymbol{v}_{i}-\boldsymbol{v}_{j}\right)}{\rho_{i} \rho_{j}}\left(\frac{1}{r_{i j}} \frac{\partial W_{i j}}{\partial r_{i}}\right) .
\end{aligned}
$$

在式(10)右边的第一项(压力项)中, 粒子 $i$ 与 $j$ 的密度 取决于其邻域的粒子分布状态, 而权函数梯度也仅 与两粒子的相对位置有关. 因此, 在特定的粒子分布 状况下, 压力项完全取决于刚度参数 $K$. 事实上, 压 力项所对应的粒子间作用力, 在物理上类似于实体 颗粒的法向作用力. 当 SPH 粒子相互重叠时, 粒子 密度以及彼此间的法向排斥力也随之快速增大. 实 体颗粒间的弹性力确实与相互重叠量具有某种形式 的正相关性, 如物理上较为合理的赫兹模型或离散 单元法(discrete element method, DEM)中广泛应用的 线弹性模型 ${ }^{[37,38]}$. SPH 粒子的刚度参数、赫兹模型的 杨氏模量以及 DEM 的刚度系数本质上都是以类似的 方式表征粒子间排斥力的强度系数. 因此, 本文将 SPH 刚度参数对流动行为的影响理解为 “弹性效应”.

另一方面, 式(10)的最后一项(黏性项)揭示, SPH 粒子的黏性效应正比于流体的动力黏度. 在式(10)所 对应的系统中, SPH 粒子间的黏性效应(耗散性质)与 弹性效应(近似守恒)相互强烈耦合. 事实上，耗散(不 可逆)与守恒 (可逆)过程在各种非平衡系统中非常常 见, Grmela 和 Öttinger ${ }^{[39]}$ 甚至提出了一个通用的耦合 理论. 基于流体黏性应力的表达式, SPH 中黏性效应 相对弹性效应的支配程度可以用如下无量纲数表示

$$
\frac{v_{0} \dot{\gamma}}{K}=\frac{v_{0} \rho \dot{\gamma}}{K \rho}=\frac{\mu_{0} \dot{\gamma}}{K \rho}=\frac{\text { 黏性效应 }}{\text { 弹性效应 }} \text {, }
$$

式中 $v_{0}$ 是流体的运动黏度, $\dot{\gamma}$ 是剪切率. 我们将这个 无量纲数称为“称弹效应比”, 下面章节将通过 Couette 流模拟考察该无量纲数对系统性质的影响.

\section{2 结果与讨论}

二维 Couette 流(坚直方向)模拟基于之前研究的 计算区域 ${ }^{[30]}$. Couette 流这个经典问题是指黏性流体 在两块无限长的平行平板间的流动现象, 其中一块 相对另一块运动. 如图 2 所示, 把流体区域分别向左 右延伸一定长度 (权函数的有效范围, 五次样条函数 为 $3 h$ ), 从而得到边壁区域. 系统初始状态的设置方 法为：首先在整个计算区域以六边形格子的方式均 匀紧密排布相等质量的粒子, 然后再把那些位于边 


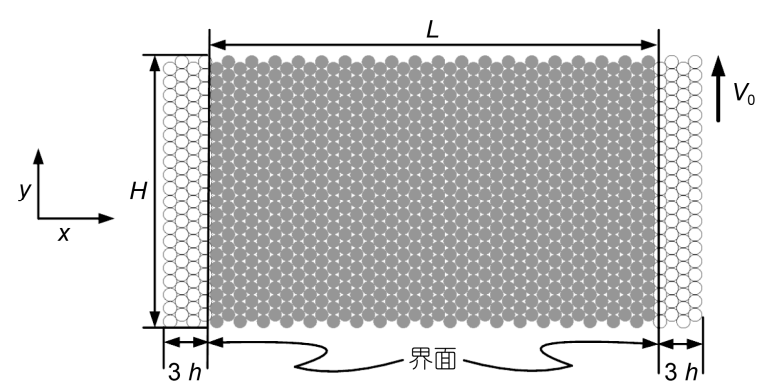

图 2 Couette 流的 SPH 模拟 ${ }^{[30]}$

壁内部的粒子指定为边界粒子(图中分别用实心圆与 空心圆表示流体粒子与边界粒子). 右边壁粒子以恒 定速度 $V_{0}$ 向上运动, 流动方向上 $(y$ 方向)采用周期边 界条件. 为获得均匀剪切流, 本文忽略重力.

在我们的算例中, 两板间距为 $1.0 \times 10^{-3} \mathrm{~m}$, 流体 密度为 $1.0 \times 10^{3} \mathrm{~kg} / \mathrm{m}^{3}$, 运动黏度为 $2.0 \times 10^{-6} \mathrm{~m}^{2} / \mathrm{s}$. 为 方便起见, 后续物理量全部用无量纲量表示, 量纲标 准分别基于权函数的有效范围 $\left(1.0 \times 10^{-4} \mathrm{~m}\right)$ 、粒子质 量 $\left(7.826 \times 10^{-11} \mathrm{~kg}\right.$ )及流体运动黏度(对应于时间量纲 $5.0 \times 10^{-3} \mathrm{~s}$ ). 经过量纲转化后, 流体的密度与动力黏 度皆为 12.78 , 两板间距为 $L=10.0$, 板长为 $H=6.0$. 粒 子的初始间距为 0.3 , 时间积分方法采用显式欧拉算 法，时间步长为 0.02 .

\section{1 速度偏差}

针对 Couette 流几个不同时刻的切向速度 $\left(\boldsymbol{v}_{y}\right)$ 剖 面, 图 3 比较了 SPH 模拟结果与相应牛顿流体的级数 解 ${ }^{[31]}$, 其中右边壁速度为 $V_{0}=0.2$, 刚度参数为 $K=$ 1.625 (黏弹效应比 $v_{0} \dot{\gamma} / K=1.231 \times 10^{-2}$ ). SPH 的速度场 几乎与级数解显示了完全一致的演化过程, 两者最 终皆呈现线性分布状态. 以最右端的流体粒子为例, $\mathrm{SPH}$ 模拟的速度误差仅为 $0.035 \%$, 这充分证明了该 算例的计算精度. 此外, 一个最右端流体粒子所受右 边壁的切向力 ( $y$ 方向), 几乎快速地在 50 个时间步内 从最初的 3.4725 下降到 0.0604 , 而且稳态振幅仅为 $1.455 \times 10^{-3}$. 同时, 该粒子所受右边壁的法向力 $(x$ 方 向)围绕 4.905 振荡, 振幅为 $1.969 \times 10^{-3}$, 只占切向力 的 3\%. 总之, 相对于往往导致压力场较大振荡的边 界力方法 ${ }^{[3]}$, 使用无滑移边界条件将获得更加可靠的 结果. 另外, 为避免 SPH 粒子在初始阶段急剧加速 所导致的不稳定性，后续算例的右边壁速度将在 $1.0 \times 10^{4}$ 的时间内从零逐渐线性增加到设定值.

在刚度参数 $K=6.5$ 时, 通过不断改变右边壁速

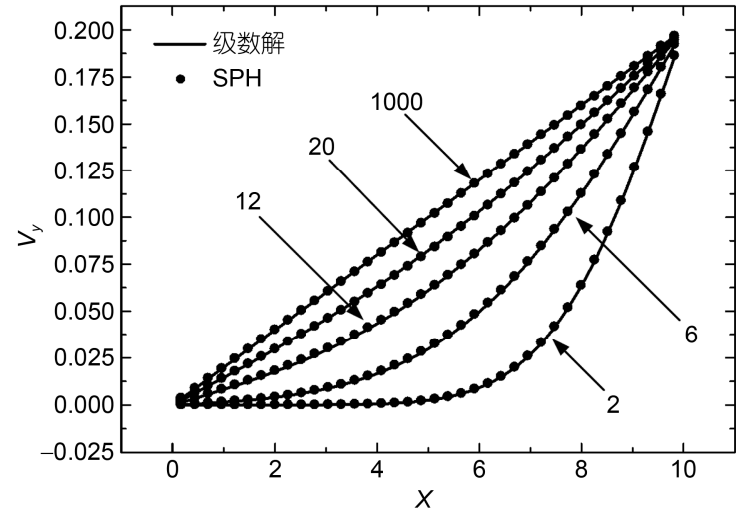

图 3 Couette 流模拟结果与级数解的比较

度, 考察不同黏弹效应比对 SPH 粒子动力学行为的 影响. 研究发现, 稳态下的速度剖面围绕线性分布状 态振荡. 振幅随着 $v_{0} \dot{\gamma} / K$ 的增大而逐渐减小, 而且当 $v_{0} \dot{\gamma} / K$ 足够大时, 它几乎接近于零. 基于我们的前期 工作 ${ }^{[30]}$, 图 4 进一步给出了 $V_{0}=0.0 、 2.5 \times 10^{-2}$ 与 0.4 时的典型模拟结果, 它们分别对应于 $v_{0} \dot{\gamma} / K=0.0$ (a)、 $3.846 \times 10^{-4}$ (b) 与 $6.154 \times 10^{-3}$ (c). 计算结果显示, SPH 粒子的速度剖面与排布方式随着无量纲数 $v_{0} \dot{\gamma} / K$ 的 增大而不断变化. 若系统完全由弹性效应支配(图 4(a)), 所有粒子将在均匀排布方式下以相等速度运 动 (极限状态是完全静止); 若基本由黏性效应支配 (图 4(c)), 系统将呈现线性速度剖面与基本无序的粒 子分布状态. 图 4(b)是上述两种情形的中间状况, 其 中的坚直线分隔了系统的不同状态层，而折线的倾 斜与水平部分分别对应于粒子的六边形与四边形排 布方式. 图 4(d)进一步放大了图 4(b)中的方形区域, 它清楚地显示速度剖面在相当程度上偏离了理论上 的线性分布状态. 在速度剖面的平台部分,粒子以六 边形格子的形式排布; 而在速度剖面的倾斜部分, 粒 子以四边形格子的形式排布. 事实上, 由于 SPH 模 拟基本保证了流体的不可压缩性，动量方程(式(10)) 的黏性项基本由速度剖面决定. 同时, 如本文第 1.3 节所述, 在给定刚度参数下, 弹性效应完全取决于粒 子的分布状态. 因此, SPH 粒子在稳态下的速度剖面 与排布方式之间的特殊对应关系，本质上是黏性效 应与弹性效应相互平衡的外在反映.

鉴于速度剖面在较小 $v_{0} \dot{\gamma} / K$ 下不容忽略的计算 误差, 传统 SPH 方法的数值精度本质上与具体问题 以及相应的模型参数相关. 只有当 $v_{0} \dot{\gamma} / K$ 足够大时, 模拟才能近似得到理想的牛顿流体行为. 因此, 在某 


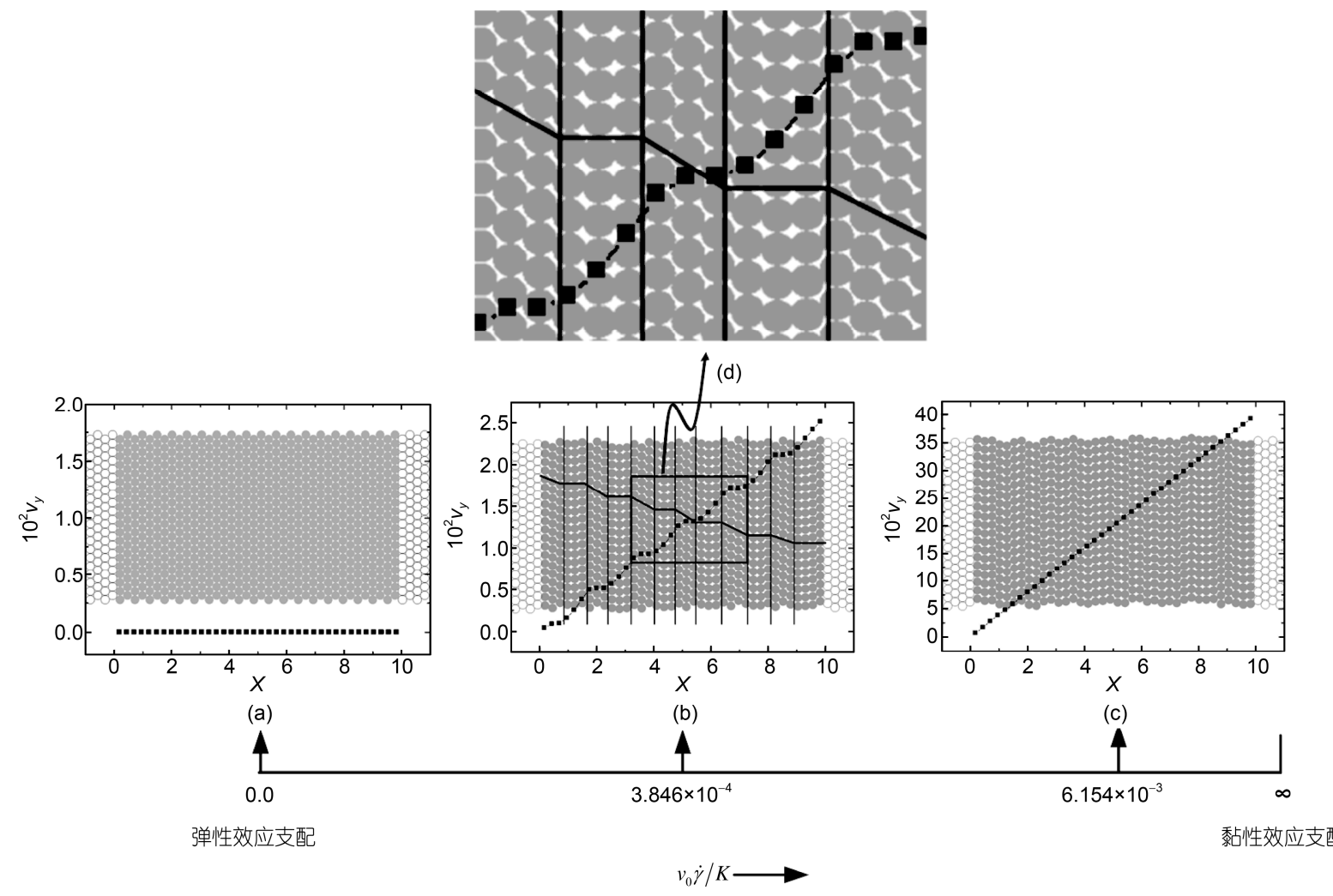

图 4 粒子的速度剖面与排布方式随黏弹效应比的变化关系 ${ }^{[30]}$

(a) $v_{0} \dot{\gamma} / K=0$; (b) $3.846 \times 10^{-4}$; (c) $6.154 \times 10^{-3}$; (d) (b)中方形区域的放大

种程度上, 该无量纲数可作为评估 SPH 模拟结果相 对牛顿流体偏差程度的一个判据. 此外, $v_{0} \dot{\gamma} / K$ 可通 过如下方式与马赫数关联

$$
\frac{v_{0} \dot{\gamma}}{K}=\frac{v_{0}}{K}\left(\frac{V_{\max }}{L}\right)=\frac{v_{0}}{L K}\left(\mathrm{MaK}^{0.5}\right)=\frac{v_{0}}{L K^{0.5}} \mathrm{Ma} .
$$

如式(12)所示, 由于马赫数要足够小才能保证流体的 不可压缩性, 因此在特定的刚度参数下, $v_{0} \dot{\gamma} / K$ 具有 相当有限的取值范围.

\section{2 有效黏度}

在不同刚度参数与剪切率下, 进一步考察黏弹 效应比这个无量纲数对 SPH 粒子有效黏度的影响. 通过统计右边壁施加在流体粒子上的切向力 $\tau$, 即可 根据表达式 $\tau / \dot{\gamma}$ 计算有效黏度 $\mu$. 事实上，稳态下的 切向力也是围绕某一特定值振荡, 因此需要基于足 够长的时间, 获得可靠的统计平均值. 图 5 给出了有 效黏度与黏弹效应比的关系, 其中不同刚度参数的 曲线几乎完全重合. 这说明有效黏度取决于无量纲 数 $v_{0} \dot{\gamma} / K$, 而不是刚度参数或剪切率. 同时, 有效黏

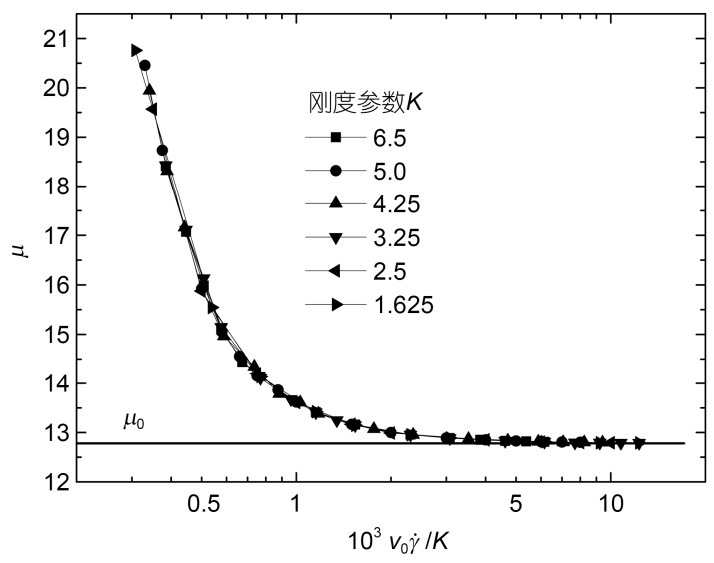

图 5 不同刚度参数下有效黏度随黏弹效应比的变化

度随着 $v_{0} \dot{\gamma} / K$ 的增大而快速下降, 并且当 $v_{0} \dot{\gamma} / K$ 足 够大时, 它几乎等于名义黏度 $\mu_{0}$ (图中标注). 因此, 为精确模拟牛顿流体行为, 保证足够大的黏弹效应 比至关重要.

另一方面, 通常认为若采用足够数量的流体粒 子, SPH 模拟将具有较高的计算精度. 因此, 在 $K=6.5$ 下，通过不断改变剪切率，进一步考察不同粒 
子数下有效黏度随黏弹效应比的变化关系. 事实上, 初始粒子间距、光滑长度及粒子质量皆随着粒子数的 增加而不断减小, 但这里的计算结果都基于本文的 量纲标准进行了转换. 如图 6 所示, 不同粒子数(包括 $760,1710,3080$ 以及 4800 )所对应的有效黏度皆随着 $v_{0} \dot{\gamma} / K$ 的增大而减小, 而且当 $v_{0} \dot{\gamma} / K$ 足够大时, 它们 都非常接近于名义黏度 $\mu_{0}$ (图中标注). 同时, 虽然在 相对较小的 $v_{0} \dot{\gamma} / K$ 下, 有效黏度随着粒子数的增多 而显著降低, 但当 $v_{0} \dot{\gamma} / K$ 大到一定程度后(如 $\left.7.692 \times 10^{-4}\right)$, 它们彼此间的差异愈发变得不明显. 总 之, 当 $v_{0} \dot{\gamma} / K$ 足够大时, 基于较少的粒子数 $(n=760)$ 即可获得良好的计算结果, 粒子数的进一步增多并 不能显著提高精度. 事实上, 如图 6 所示, 由于某种 数值误差, 粒子数 3080 与 4800 所对应的有效黏度甚 至稍微偏离名义黏度.

为保证足够大的黏弹效应比, 相对某一特定剪 切率的刚度参数必须足够小. 然而, 为保证流体的不 可压缩性(较小的马赫数), 刚度参数又必须足够大. 因此, 刚度参数的选择必须同时考虑这两个竞争性 因素. 鉴于不可压缩流的最大相对密度变化率为 $\delta=$ $0.03^{[31]}$, 刚度参数取为 $30 V_{\text {max }}^{2}$ 左右在某种程度上比 较合适. 此外, 把黏弹效应比中的 $K$ 与 $\dot{\gamma}$ 分别用式(7) 与 $V_{\max } / L$ 替换, 可进一步将其改写为

$$
\frac{v_{0} \dot{\gamma}}{K}=v_{0}\left(\frac{\mathrm{Ma}}{V_{\text {max }}}\right)^{2}\left(\frac{V_{\text {max }}}{L}\right)=\frac{v_{0}}{V_{\text {max }} L} \mathrm{Ma}^{2}=\frac{\mathrm{Ma}^{2}}{\mathrm{Re}} .
$$

如式(13)所示, 相对某一特定马赫数, $v_{0} \dot{\gamma} / K$ 将直接 反比于雷诺数 Re. 因此, 传统 SPH 方法本质上更适 合模拟对应于较大黏弹效应比的低雷诺数流动, 此

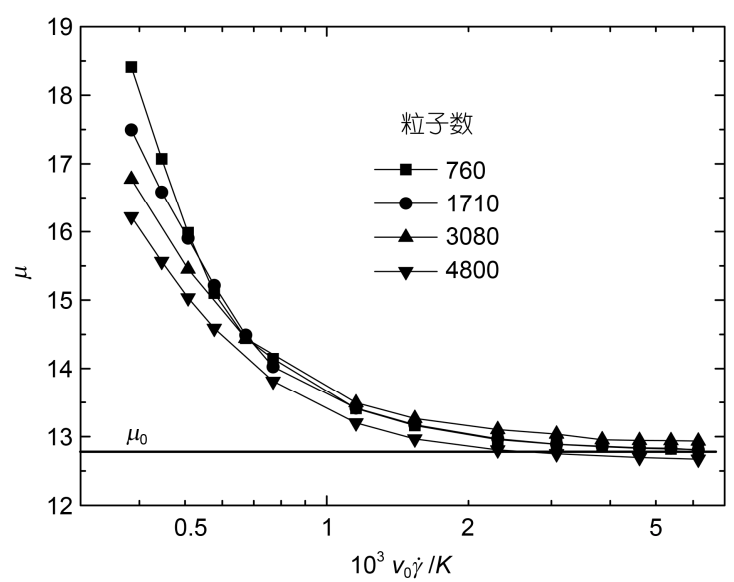

图 6 不同流体粒子数下有效黏度随黏弹效应比的变化
时才能保证较高的计算精度. 根据图 4 中速度剖面的 变化趋势以及图 5 和 6 中有效黏度的变化趋势, 初步 判断当 $v_{0} \dot{\gamma} / K$ 大于 $3 \times 10^{-3}$ 时, 模拟结果将较好地接 近于牛顿流体. 所以, 传统 SPH 方法存在一个具有 较高模拟精度的临界雷诺数

$$
\mathrm{Re}=\frac{\mathrm{Ma}^{2}}{v_{0} \dot{\gamma} / K}<\frac{\mathrm{Ma}^{2}}{3 \times 10^{-3}}<\frac{0.03}{3 \times 10^{-3}}=10 .
$$

\section{3 能量耗散}

进一步考察 SPH 粒子系统的能量耗散. 针对动 力黏度为 $\mu_{0}$ 的不可压牛顿流体, 单位体积的黏性耗 散 $\phi($ 此后称之为名义黏性耗散) 在二维下的定义为

$$
\phi=2 \mu_{0}\left[\left(\frac{\partial v^{x}}{\partial x}\right)^{2}+\left(\frac{\partial v^{y}}{\partial y}\right)^{2}\right]+\mu_{0}\left(\frac{\partial v^{y}}{\partial x}+\frac{\partial v^{x}}{\partial y}\right)^{2},
$$

式中 $v^{x}$ 与 $v^{y}$ 分别是流体在 $x$ 与 $y$ 方向上的速度. 根据 $\mathrm{SPH}$ 的基本理论，基于粒子 $i$ 的局部名义黏性耗散可 通过下式计算

$$
\begin{aligned}
\phi_{i}= & \frac{\mu_{0}}{\rho_{i}^{2}}\left[2\left(\sum_{j} m_{j} v_{i j}^{x} \nabla_{i} W_{i j}^{x}\right)^{2}+2\left(\sum_{j} m_{j} v_{i j}^{y} \nabla_{i} W_{i j}^{y}\right)^{2}\right. \\
& \left.+\left(\sum_{j} m_{j} v_{i j}^{y} \nabla_{i} W_{i j}^{x}+\sum_{j} m_{j} v_{i j}^{x} \nabla_{i} W_{i j}^{y}\right)^{2}\right],
\end{aligned}
$$

式中 $v_{i j}^{x}$ 与 $v_{i j}^{y}$ 分别是粒子 $i$ 与 $j$ 在 $x$ 与 $y$ 方向上的相对 速度; $\nabla_{i} W_{i j}^{x}$ 与 $\nabla_{i} W_{i j}^{y}$ 分别表示权函数在 $x$ 与 $y$ 方向上 相对坐标 $\boldsymbol{r}_{i}$ 的梯度.

对所有流体粒子的局部黏性耗散进行累加, 进 一步获得整个系统的名义黏性耗散 $\phi_{\mathrm{vis}}$, 具体公式为

$$
\phi_{\mathrm{vis}}=\sum_{i} \frac{m_{i}}{\rho_{i}} \phi_{i} .
$$

此外, 系统在稳态下的总耗散 $\phi_{\text {total }}$ 可以简单地通过如 下能量衡算的方式计算

$$
\phi_{\text {total }}=F V_{0},
$$

式中 $F$ 是右边壁所承受的切向力.

在右边壁速度为 $V_{0}=2.5 \times 10^{-2}$ 时, 通过不断改变 刚度参数, 图 7 给出了系统的名义黏性耗散与总耗散 随黏弹效应比的变化关系. 为便于比较, 图中也标注 了相应牛顿流体 (动力黏度为 12.78)的理论黏性耗散 $\left(4.793 \times 10^{-3}\right)$. 由于速度剖面的振荡现象, 图 7 的数据 都是基于可靠的统计平均值. 当 $v_{0} \dot{\gamma} / K$ 相对较小时, 总耗散较大地偏离名义黏性耗散, 而且两者都显著 


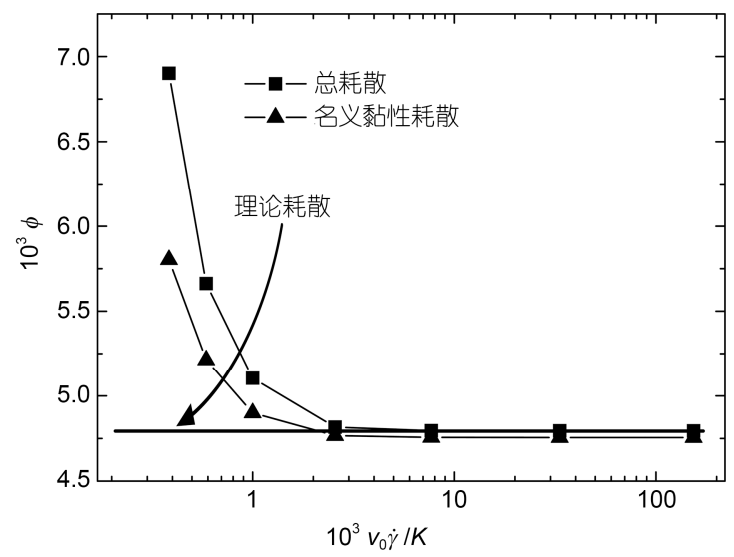

图 7 名义黏性耗散与总耗散随㩯弹效应比的变化

地大于理论耗散. 随着 $v_{0} \dot{\gamma} / K$ 的进一步增大, 总耗散 与名义㯟性耗散都以强烈的非线性方式(类似于指数 函数)快速下降. 当 $v_{0} \dot{\gamma} / K$ 足够大时, 名义黏性耗散 与总耗散都几乎等于理论耗散. 本质上, 极大的 $v_{0} \dot{\gamma} / K$ 表明系统中黏性效应占支配作用, 此时可较 好地模拟牛顿流体, 从而对应于理论上的最小黏性 耗散. 在相对较小的 $v_{0} \dot{\gamma} / K$ 下, 弹性效应发挥重要影 响, 此时的系统将显著地偏离预期的牛顿流体, 从而 导致较大的名义黏性耗散. 然而, SPH 模拟在极大的 刚度参数下将变得异常不稳定, 因此弹性效应绝对 支配的状态实际上是不存在的.

事实上, 早期研究 ${ }^{[40]}$ 已发现, 无黏欧拉流体的 SPH 模拟存在某种内在耗散. 本文中名义黏性耗散 与总耗散间的差异, 揭示了内在耗散也存在于不可压 牛顿流体的 SPH 模拟, 同时进一步验证了附加的人 工黏度, 即图 5 中有效黏度相对于名义黏度的偏差量.
此外, 我们也考虑了速度 Verlet 积分算法, 但结果几 乎一致. 总之, 这些非物理现象本质上皆可归结于 SPH 方法的离散思想. 连续的流体仅被离散为非常 有限数目的粒子, 粒子在计算中的不规则分布状态 导致了较低的数值精度. 对于有限的粒子数, 此计算 误差难以避免, 而且误差量基本取决于系统的黏弹 效应比.

\section{3 结论}

基于对传统 SPH 方法中粒子物理性质的分析, 本文提出了一个表征系统中黏性效应相对弹性效应 支配强度的无量纲数 $v_{0} \dot{\gamma} / K$. 通过模拟二维 Couette 流, 研究发现速度剖面、有效黏度以及总耗散相对各 自理论值的偏差量皆随着 $v_{0} \dot{\gamma} / K$ 的增大而减小. 在 某种意义上, 该无量纲数可用于评估 SPH 模拟结果 相对牛顿流体的偏差程度. 由于马赫数与黏弹效应 比的双重限制, 传统 SPH 方法具有一定的适用范围, 即存在一个临界雷诺数. 只有当系统的雷诺数低于 这个临界数时, 才可以利用合适的模型参数精确模 拟牛顿流体.

同时, 对于高雷诺数流动, 有必要通过额外的校 正措施消除 SPH 粒子的弹性效应所导致的计算误差. 除了引言中提到的各种复杂人工改进方案, 从无量 纲数的角度修正传统 SPH 方法可能是一个颇有意义 的思路. 事实上, 当黏弹效应比相对较小时, SPH 粒 子本质上对应于某种特殊的非牛顿流体 ${ }^{[30]}$. 另外, 本 文只是初步探讨了这个无量纲数的物理意义, 该无 量纲数对其他复杂流动的普适性也有待进一步验证.

\section{参考文献}

1 Gingold R A, Monaghan J J. Smoothed particle hydrodynamics: Theory and application to non-spherical stars. Mon Not R Astron Soc, 1977, 181: 375-389

2 Lucy L B. A numerical approach to the testing of the fission hypothesis. Astron J, 1977, 82: 1013-1024

3 Monaghan J J. Simulating free surface flows with SPH. J Comput Phys, 1994, 110: 399-406

4 Shao S, Lo E Y M. Incompressible SPH method for simulating Newtonian and non-Newtonian flows with a free surface. Adv Water Resour, 2003, 26: 787-800

5 Zhou G, Ge W, Li B, et al. SPH simulation of selective withdrawal from microcavity. Microfluid Nanofluid, 2013, doi: 10.1007/s10404013-1165-1

6 Zhang M. Simulation of surface tension in 2D and 3D with smoothed particle hydrodynamics method. J Comput Phys, 2010, 229: 7238-7259

7 Xu X, Ouyang J, Jiang T, et al. Numerical simulation of 3D-unsteady viscoelastic free surface flows by improved smoothed particle hydrodynamics method. J Non-Newton Fluid Mech, 2012, 177-178: 109-120

$8 \mathrm{Xu} \mathrm{X,} \mathrm{Ouyang} \mathrm{J,} \mathrm{Yang} \mathrm{B,} \mathrm{et} \mathrm{al.} \mathrm{SPH} \mathrm{simulations} \mathrm{of} \mathrm{three-dimensional} \mathrm{non-Newtonian} \mathrm{free} \mathrm{surface} \mathrm{flows.} \mathrm{Comput} \mathrm{Methods} \mathrm{Appl} \mathrm{Mech}$ Engrg, 2013, 256: 101-116 
9 Shao S. Incompressible SPH simulation of wave breaking and overtopping with turbulence modeling. Int J Numer Methods Fluids, 2006, 50: $591-621$

10 Violeau D, Issa R. Numerical modelling of complex turbulent free-surface flows with the SPH method: An overview. Int J Numer Methods Fluids, 2007, 53: 277-304

11 Rafiee A, Thiagarajan K P. An SPH projection method for simulating fluid-hypoelastic structure interaction. Comput Methods Appl Mech Engrg, 2009, 198: 2785-2795

12 Li S, Liu W K. Meshfree and particle methods and their applications. Appl Mech Rev, 2002, 55: 1-34

13 Belytschko T, Krongauz Y, Dolbow J, et al. On the completeness of meshfree particle methods. Int J Numer Methods Eng, 1998, 43: 785-819

14 Liu M B, Liu G R. Restoring particle consistency in smoothed particle hydrodynamics. Appl Num Math, 2006, 56: 19-36

15 Koumoutsakos P. Multiscale flow simulations using particles. Annu Rev Fluid Mech, 2005, 37: 457-487

16 Liu W K, Jun S, Zhang Y F. Reproducing kernel particle methods. Int J Numer Methods Fluids, 1995, 20: 1081-1106

17 Chen J K, Beraun J E, Carney T C. A corrective smoothed particle method for boundary value problems in heat conductions. Int J Numer Methods Eng, 1999, 46: 231-252

18 Liu M B, Xie W P, Liu G R. Modeling incompressible flows using a finite particle method. Appl Math Model, 2005, 29: 1252-1270

19 Jiang T, Ouyang J, Ren J, et al. A mixed corrected symmetric SPH (MC-SSPH) method for computational dynamic problems. Comp Phys Comm, 2012, 183: 50-62

20 Dilts G A. Moving-least-squares-particle hydrodynamics-I. Consistency and stability. Int J Numer Methods Eng, 1999, 44: 1115-1155

21 Chaniotis A K, Poulikakos D, Koumoutsakos P. Remeshed smooth particle hydrodynamics for the simulation of viscous and heat conducting flows. J Comput Phys, 2002, 182: 67-90

22 Monaghan J J. Smoothed particle hydrodynamics. Rep Prog Phys, 2005, 68: 1703-1759

23 Posch H A, Hoover W G, Kum O. Steady-state shear flows via nonequilibrium molecular dynamics and smooth-particle applied mechanics. Phys Rev E, 1995, 52: 1711-1720

24 Hoover W G, Hess S. Equilibrium and nonequilibrium thermomechanics for an effective pair poterntial used in smooth particle applied mechanics. Physica A, 1996, 231: 425-438

25 Español P, Revenga M. Smoothed dissipative particle dynamics. Phys Rev E, 2003, 67: 026705

26 Tartakovsky A, Meakin P. Modeling of surface tension and contact angles with smoothed particle hydrodynamics. Phys Rev E, 2005, 72: 026301

27 Tartakovsky A M, Meakin P. Pore scale modeling of immiscible and miscible fluid flows using smoothed particle hydrodynamics. Adv Water Resour, 2006, 29: 1464-1478

28 Zhou G, Ge W, Li J. A revised surface tension model for macro-scale particle methods. Powder Technol, 2008, 183: 21-26

29 Zhou G, Chen Z, Ge W, et al. SPH simulation of oil displacement in cavity-fracture structures. Chem Eng Sci, 2010, 65: 3363-3371

30 Zhou G, Ge W, Li J. Smoothed particles as a non-Newtonian fluid: A case study in Couette flow. Chem Eng Sci, 2010, 65: 2258-2262

31 Morris J P, Fox P J, Zhu Y. Modeling low Reynolds number incompressible flows using SPH. J Comput Phys, 1997, 136: 214-226

32 Sigalotti L D G, Klapp J, Sira E, et al. SPH simulations of time-dependent Poiseuille flow at low Reynolds numbers. J Comput Phys, 2003, 191: $622-638$

33 Colagrossi A, Landrini M. Numerical simulation of interfacial flows by smoothed particle hydrodynamics. J Comput Phys, 2003, 191: 448-475

$34 \mathrm{Hu}$ X Y, Adams N A. A multi-phase SPH method for macroscopic and mesoscopic flows. J Comput Phys, 2006, 213: 844-861

35 Ellero M, Tanner R I. SPH simulations of transient viscoelastic flows at low Reynolds number. J Non-Newton Fluid Mech, 2005, 132: $61-72$

36 Zhu H, Martys N S, Ferraris C, et al. A numerical study of the flow of Bingham-like fluids in two-dimensional vane and cylinder rheometers using a smoothed particle hydrodynamics (SPH) based method. J Non-Newton Fluid Mech, 2010, 165: 362-375

37 Zhu H, Zhou Z, Yang R, et al. Discrete particle simulation of particulate systems: Theoretical developments. Chem Eng Sci, 2007, 62: 3378-3396

38 Sun Q, Wang G, Hu K. Some open problems in granular matter mechanics. Prog Nat Sci, 2009, 19: 523-529

39 Grmela M, Öttinger H C. Dynamics and thermodynamics of complex fluids. I. Development of a general formalism. Phys Rev E, 1997, 56: $6620-6632$

40 Hoover W G, Pierce T G, Hoover C G, et al. Molecular dynamics, smoothed-particle applied mechanics, and irreversibility. Comput Math Appl, 1994, 28: 155-174 